\title{
Damage test for International Linear Collider positron generation target at KEKB
}

\author{
M. Kuriki, ${ }^{*}$ T. Mimashi, K. Saito, M. Kikuchi, and T. Kamitani \\ High Energy Accelerator Research Organization, Oho 1-1, Postal code 305-0801, Tsukuba, Ibaraki, Japan
}

(Received 19 March 2006; published 26 July 2006)

\begin{abstract}
ILC (International Linear Collider) is aiming to conduct electron-positron collisions at $1 \mathrm{TeV}$ center-ofmass energy. One bunch train will contain up to $28003.2 \mathrm{nC}$ bunches with a $308 \mathrm{~ns}$ bunch spacing or 5600 $1.6 \mathrm{nC}$ bunches with a $154 \mathrm{~ns}$ spacing. The bunch-train length will be $0.9 \mathrm{~ms}$. Because of this extremely large amount of beam in a train, serious damage to a positron production target driven by $6 \mathrm{GeV}$ incident electron beam is of concern. As the ILC positron source, several different methods have been proposed. The target hardness is a key point concerning the selection. In this article, we report on a test experiment to examine the target hardness by using a stored electron beam in KEKB HER (High Energy Ring). The project name is IPPAK (ILC Positron Project At KEKB). By manipulating the abort kicker, a condition similar to that of the ILC positron production target can be reproduced. The experiment was carried out on June 29 and 30,2005. The target was seriously damaged under the heaviest condition (KEKB mode), but the damage was less and nothing for those of the ILC mode. Possible impacts to the ILC positron production scheme are also discussed.
\end{abstract}

DOI: 10.1103/PhysRevSTAB.9.071001

PACS numbers: 07.77.Ka, 61.80.Fe, 29.25.- t

\section{INTRODUCTION}

The ILC (International Linear Collider) [1] is a linear collider project promoted by ICFA (International Committee for Future Accelerators) [2]. In the summer of 2004, ICFA decided to promote the ILC project based on superconducting technology according to a report of the ITRP (International Technology Recommendation Panel) [3].

In the superconducting accelerator, the pulse length will be much longer than that of the normal conducting accelerator for efficient acceleration. In the ILC case, one rf pulse length will be $0.9 \mathrm{~ms}$. The system will be operated at $5 \mathrm{~Hz}$. To achieve the required luminosity in the range of $2.0 \times 10^{38} \mathrm{~m}^{-2} \mathrm{~s}^{-1}, 28003.2 \mathrm{nC}$ bunches or $56001.6 \mathrm{nC}$ bunches are to be filled in a train with a 308 or $154 \mathrm{~ns}$ bunch spacing, resulting in a $0.9 \mathrm{~ms}$ length bunch train with a $10 \mathrm{~mA}$ average current.

A conventional method used to produce a positron beam is the pair-creation process in a heavy material driven by a high-energy electron beam. In the target, an electromagnetic shower grows up while generating a mixed flux of electrons, positrons, and gammas. From the flux, a positron beam is selected by bending magnets.

If we produce the ILC positron bunch train by this conventional method, the target will be broken immediately because more than $9 \mathrm{~kW}$ of the beam energy will be concentrated in a small spot (typically several $\mathrm{mm}^{2}$ ) within $0.9 \mathrm{~ms}$. According to an experiment carried out at SLAC [4], the damage threshold for a single bunch was $320 \mathrm{~J} / \mathrm{mm}^{2}$. The examined target material was $\mathrm{W}(75) \operatorname{Re}(25)$ alloy, which has been used as a SLAC Linear Collider positron production target. If we assume

*Electronic address: masao.kuriki@kek.jp a $1 \mathrm{~mm}^{2}$ spot size and a $6 \mathrm{GeV}$ drive beam giving the positron yield per incident electron to be 1.0, the energy deposit in the ILC positron production target will be $2800 \times 6 \times 3.2=53760 \mathrm{~J} / \mathrm{mm}^{2}$, which is clearly much higher than the threshold.

A totally new method to generate a positron beam was proposed by the TESLA collaboration [5]. In this method, high-energy gamma rays (up to $26 \mathrm{MeV}$ ), generated by a $70 \mathrm{~m}$ planar undulator with a $250 \mathrm{GeV}$ electron beam, are injected in the production target, which has a smaller thickness (0.4 radiation length), resulting in less damage. Because it is very hard to implement a $250 \mathrm{GeV}$ electron linac dedicated to positron production, the electron beam before collisions is used to generate high-energy photons. This method is, however, totally new and has never been built. It is very difficult to examine the feasibility prior to a real ILC machine with a sufficient level. One might be concerned about the total availability of ILC because this scheme relies on interdependent systems of electrons and positrons. Electron-beam quality degradation, e.g., emittance growth, could be a serious problem, since the electron beam passes the undulator section before the interaction point.

Another new method to generate the ILC positron beam was proposed by Omori [6] at the second ILC Workshop at Snowmass, Colorado. In this method, high-energy gamma rays are generated by Compton scattering between the laser photon and electron beam. Since the positron intensity from one collision is much less than the required value, many positron bunches are accumulated (stacked) in the damping ring. As a by-product, the target damage is much less than the others, but many critical devices have to be developed to realize this system.

The conventional method is therefore the most viable option as long as the target damage problem is resolved. 
One way to avoid target breakage in the conventional method is to rotate the target, and thus spreading widely the energy deposition. Technically, a rotation speed of 3600 RPM with $1 \mathrm{~m}$ radius, which corresponds to $360 \mathrm{~m} / \mathrm{s}$ tangential speed, is possible [7]. This rotation speed was decided to prevent a fatigue effect on the target material (W-Re alloy).

The threshold for the target breakage observed in SLAC was obtained with a condition of single-bunch injection. In ILC, however, the beam bunch comes with a relatively large bunch spacing of $308 \mathrm{~ns}$. The power defined as the energy per time is much different for the ILC and SLAC cases. There may be a possibility that the threshold depends on the duration of the incident beam, and thus the threshold of target breakage is larger for the ILC case. In that case, the maximum target rotation speed to prevent target damage can be lowered. Even if any fatigue damage occurs with this lower rotation speed, the system is still operable as long as mean time between failure causing the fatigue damage is long enough, e.g., one year.

Lowering the target speed has many advantages. With a fixed rotation speed, the target radius can be smaller. The target station and the area will be smaller and cost effective. The lowered rotation also eases the conditions of the technical design. For example, the target is placed in front of a solenoid magnet, which produces a strong magnetic field of more than $5 \mathrm{~T}$, to capture generated positrons. Fast rotation in such a strong magnetic field causes an unstable motion due to the eddy current. This problem can be relaxed.

In the ILC Positron Project At KEKB (IPPAK) experiment, the stored beam of KEKB High Energy Ring (HER) $[8,9]$ is injected into a test material placed inside the beam dump. By manipulating the beam-fill pattern and abort kicker, a beam condition similar to the ILC positron production drive beam (energy density of $1410 \mathrm{~J} / \mathrm{mm}^{2}$ ) can be reproduced. The IPPAK experiment can demonstrate the possible damage on the ILC positron production target and examine the feasibility of the conventional method of positron production for ILC.

\section{SETUP}

The experiment was carried out at KEKB HER [8,9]. A beam-abort system was implemented to reserve radiation safety and to protect sensitive components in the BELLE detector. In beam abortion, the circulating beam is deflected by the kicker system and guided to the dump line through a Lambertson Septum magnet [10]. At the end of the dump line, a beam dump is placed to dispose the electron beam and seals the radiation.

The system has two kicker magnets for horizontal and vertical deflection, respectively. The kicker magnets are conventional window-frame type magnets with a ferrite core driven by a single power supply. Sinusoidal oscillation has been introduced on top of the horizontal kicker pulse in

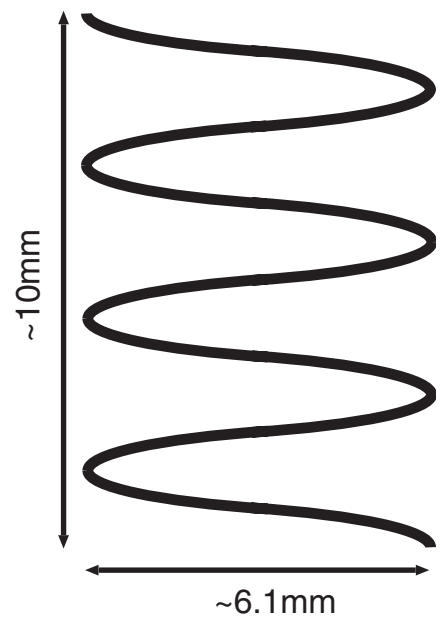

FIG. 1. A trace drawn by the aborted bunches on the beam dump in KEKB HER. This pattern is made from vertical and horizontal kicks.

order to relax the power density in the extraction window [10].

As a result of the vertical and horizontal sweeps, the aborted beam follows a semisinusoidal shape, as shown in Fig. 1.

Figure 2 shows the experimental layout of IPPAK. The test target is deeply inserted into a cave which is located on the dump wall with $600 \mathrm{~mm}$ depth. The beam dump is composed of iron, lead, and concrete. Because the beam dump has been used since KEKB operation started, the material of the dump is expected to be activated radiologically. To prevent possible radiation pollution from scratching the inner wall in the process of mounting and dismounting of the test target, the inner surface of the cave is covered by a guide pipe.

Test material is mounted in a cassette that is $74 \mathrm{~mm}$ in diameter and $200 \mathrm{~mm}$ tall. There are three purposes of the

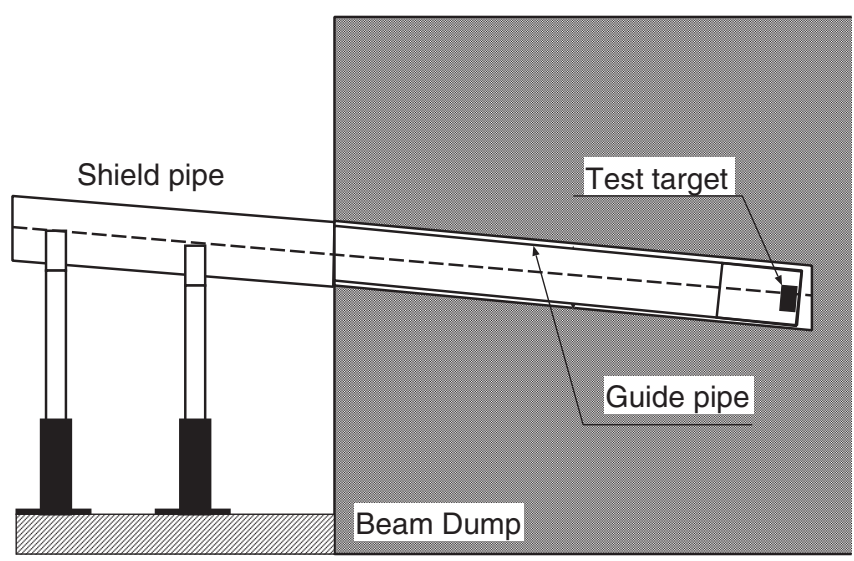

FIG. 2. Schematic view of the basic experimental setup. The test target is inserted into the end of the dump cave. To prevent any radiation pollution during the experiment (loading and unloading the target), a guide pipe is inserted to cover the radiated dump wall. 
cassette. One is to mount and unmount the test material quickly to/from the cave. It is very important to shorten the access time for efficient operation and especially for radiation safety. The second purpose of the cassette is to maintain the distance from the dosed material when handling it. As mentioned later, the radioactivity of the exposed target is not negligible. That is why it is important to maintain the distance from the material to reduce exposure. The last purpose is to precisely control the target material position.

\section{EXPERIMENTAL MODES}

Table I summarizes the characteristics of the drive beam for the ILC positron source and the KEKB HER stored beam. Because the total energy of the ILC drive beam is almost half of that of the KEKB HER stored beam, a condition equivalent to the ILC drive beam can be reproduced by manipulating the KEKB HER stored beam. The biggest difference is the pulse duration, $920 \mu$ s for ILC and only $9.1 \mu \mathrm{s}$ for KEKB HER.

If the trace shown in Fig. 1 is approximated to be a sinusoidal function ( $3.1 \mathrm{~mm}$ amplitude and $2850 \mathrm{~ns}$ revolution), the distribution of the energy deposited on the beam dump can be analyzed. The bunch spacing is 6 or $8 \mathrm{~ns}$, but let us assume here $7 \mathrm{~ns}$. The step size for each bunch, i.e., the distance between the injection points of the neighboring bunches, depends on the position in the pattern. The step size is maximized at the zero cross of the sinusoidal curve, to be $53.5 \mu \mathrm{m}$. The minimum is $7.14 \mu \mathrm{m}$ at the extremum. If the energy of a single bunch deposited in the target is distributed in a disk of $0.56 \mathrm{~mm}$ radius making $1.0 \mathrm{~mm}^{2}$ area constantly, the expected energy density accounting for the overlapped bunches can be estimated. The electron beam size at the entrance of the dump line is $0.6 \mathrm{~mm}$ horizontal and $0.3 \mathrm{~mm}$ vertical. This size is slightly increased by-passing air $4 \mathrm{~m}$ long. In addition, this spot size is further increased by developing an elecromagnetic shower in the $1.5 \mathrm{~cm} \mathrm{~W}$-Re block. A $0.56 \mathrm{~mm}$ radius on the spot size can be considered a reasonable assumption.

Table II summarizes the estimated energy density on the target. The energy density is maximized along the line connecting the center of the spots. The estimated energy density shown in this table is this maximum value. For a comparison, ILC drive beam parameters are also listed. For the ILC, target rotation with a speed of $50 \mathrm{~m} / \mathrm{s}$, giving $15.4 \mu \mathrm{m}$ displacement during $308 \mathrm{~ns}$, is assumed. To estimate the bunch overlap, it was assumed that the beam energy is constantly deposited on a $1 \mathrm{~mm}^{2}$ area, the same as in the KEKB case. With this assumption, the number of overlaps was estimated to be $1.13 /\left(15.4 \times 10^{-3}\right)=73.4$. Multiplying the energy density of one bunch $\left(19.2 \mathrm{~J} / \mathrm{mm}^{2}\right)$ by the number of overlaps, the maximum energy density along the injected beam path on the target can be estimated. The duration is the time to make this energy density on a spot, in other words, the number of overlap multiplied
TABLE I. Characters of the ILC positron driver beam and KEKB HER stored beam.

\begin{tabular}{lcc}
\hline \hline \multicolumn{1}{c}{ Item } & ILC & KEKB \\
\hline Bunch number & 2800 & 1300 \\
Beam energy & $6 \mathrm{GeV}$ & $8 \mathrm{GeV}$ \\
Bunch charge & $3.2 \mathrm{nC}$ & $10 \mathrm{nC}$ \\
Bunch spacing & $308 \mathrm{~ns}$ & 6 or $8 \mathrm{~ns}$ \\
Total duration & $862 \mu \mathrm{s}$ & $9.1 \mu \mathrm{s}$ \\
Total energy & $53760 \mathrm{~J}$ & $10400 \mathrm{~J}$ \\
\hline \hline
\end{tabular}

TABLE II. Summary of the beam parameters and expected energy deposited in the ILC drive beam and KEKB stored beam. For ILC, $50 \mathrm{~m} / \mathrm{s}$ target rotation is assumed.

\begin{tabular}{lcc}
\hline \hline \multicolumn{1}{c}{ Item } & ILC & KEKB \\
\hline Bunch charge $(\mathrm{nC})$ & 3.2 & 10 \\
Beam energy $(\mathrm{GeV})$ & 6.0 & 8.0 \\
Bunch power $(\mathrm{J})$ & 19.2 & 80 \\
Step size $(\mu \mathrm{m})$ & 15.4 & $7.14-53.5$ \\
Bunch overlap & 73.4 & $21.1-158$ \\
Energy density $\left(\mathrm{J} / \mathrm{mm}^{2}\right)$ & 1410 & $1690-12600$ \\
Duration $(\mu \mathrm{s})$ & 22.6 & $0.148-1.11$ \\
\hline \hline
\end{tabular}

by the bunch spacing. If the target rotation is faster, the step size is larger and the bunch overlap, energy density, and the duration are less.

Since the lowest energy density of KEKB is $1690 \mathrm{~J} / \mathrm{mm}^{2}$, the energy density in the ILC case, $1410 \mathrm{~J} / \mathrm{mm}^{2}$, can be reproduced by reducing the KEKB HER beam intensity with a factor of $83 \%$. The ILC condition with a faster rotating target can be reproduced by reducing the beam intensity further. Let us call this experimental mode the KEKB mode.

Although the energy density is reproduced in the KEKB mode, the duration is much different for the real ILC drive beam and the KEKB mode. To reproduce both the energy density and the power (energy flux) density, which is defined as the ratio of the energy and duration, another experimental mode, the ILC mode, is considered. By turning off the vertical kicker, the trace of the beams becomes a simple horizontal oscillation instead of a sinusoidal wave, with a $2850 \mathrm{~ns}$ revolution period. If only appropriate bunches around the zero point are filled, multiple bunches with $1425 \mathrm{~ns}$ spacing (half cycle of the oscillation) are overlaid on the same spot.

If only one bunch is filled at each zero-cross position, 7 bunches are available in total. Note that the revolution period of the KEKB ring is $10 \mu$ s. Since the energy of one bunch is $8 \mathrm{GeV} \times 10 \mathrm{nC}=80 \mathrm{~J}$, the total energy density and flux are $80 \times 7=560 \mathrm{~J} / \mathrm{mm}^{2}$ and $560 /(1.43 \times$ $6)=65.5 \mathrm{~J} / \mu \mathrm{s}$. Let us call this experimental mode ILC 1.

If we put two bunches for each zero-cross position, the total energy density and flux are $2 \times 80 \times 7=$ $1120 \mathrm{~J} / \mathrm{mm}^{2}$ and $1120 /(1.43 \times 6)=131 \mathrm{~J} / \mu \mathrm{s}$. Let us 
TABLE III. Summary of the beam parameters and expected energy density and flux in the ILC drive beam (ILC) and KEKB operated with ILC modes (ILC 1 and 2).

\begin{tabular}{lccc}
\hline \hline \multicolumn{1}{c}{ Item } & ILC & ILC1 & ILC2 \\
\hline Bunch charge $(\mathrm{nC})$ & 3.2 & 10 & 10 \\
Beam energy $(\mathrm{GeV})$ & 6.0 & 8.0 & 8.0 \\
Bunch power $(\mathrm{J})$ & 19.2 & 80.0 & 80.0 \\
Bunch overlap & 73.4 & 7.00 & 14.0 \\
Energy density $\left(\mathrm{J} / \mathrm{mm}^{2}\right)$ & 1410 & 560 & 1120 \\
Duration $(\mu \mathrm{s})$ & 22.6 & 8.55 & 8.55 \\
Energy Flux $(\mathrm{J} / \mu \mathrm{s})$ & 62.4 & 65.5 & 131 \\
\hline \hline
\end{tabular}

call this mode ILC2. The displacement of the neighbor bunches is only $53.5 \mu \mathrm{m}$, which is negligible compared to a spot size of $1 \mathrm{~mm}^{2}$.

In the ILC drive beam, the energy density and flux are estimated to be $19.2 \times 73.4=1410 \mathrm{~J} / \mathrm{mm}^{2}$ and $19.2 / 0.308=62.4 \mathrm{~J} / \mu \mathrm{s}$. Therefore, ILC modes 1 and 2 reproduce the energy flux and the energy density of the ILC drive beam, respectively. The parameters of the ILC modes are summarized in Table III.

\section{RADIATION SAFETY}

The radiation safety is a big issue in two contexts: radiation safety regulation and damage to the KEKB system.

In the first design of the target mount system, the target is placed in front of the beam dump. In this scheme, the targets are exchanged remotely without any access to the accelerator tunnel, so that we do not need any interval between the shots, which would be necessary for radiological activation decay; the experiment can be carried out quickly. Since most of the radiation flux generated from the beam interaction in the test target is distributed in the forward direction where the beam dump exists, this flux is absorbed by the dump block. According to an estimation by Tawara and Saito [11], however, the backward flux is not sizable: Particles that are backscattered in the test target, and scattered secondary at the surface of the dump block, cannot be absorbed by the dump block, and are spilled into the accelerator tunnel. The averaged radiation flux will be $1 \mathrm{E}+4$ times larger than that under the nominal dump condition. Even in that case, because the radiation flux with the nominal dump condition is much smaller compared to the limit of the safety regulation, the regulation is still satisfied.

In another context, this flux might be very serious. The tolerable radiation level to the KEKB and BELLE systems is not known, and hard to guess beforehand. By considering this system-safety issue conservatively, we finally gave up this front target mount.

In the final design, the target is mounted deeply inside of the hole on the beam dump block. In this scheme, the radiation flux to the surroundings is the same as that in the usual dump. With this configuration, IPPAK neither violates the radiation safety regulation nor damages any devices in KEKB. The cost is that the target has to be manually replaced after the experiment shots, and some radiation exposure is expected.

According to an estimation by Saito of the KEK radiation science center, the absorbed dose right after the experiment shot with the full intensity of KEKB stored beam is $3.81 \times 10^{2} \mathrm{~Sv} / \mathrm{h}$ at $1 \mathrm{~cm}$ away from the target [12]. In the estimation, a $1.5 \mathrm{~cm}$ thick W (tungsten) target, where $20 \%$ of the beam energy is deposited, was used. It decreased to $2.92 \times 10^{-1} \mathrm{~Sv} / \mathrm{h}$ one hour later and $9.32 \times$ $10^{-4} \mathrm{~Sv} / \mathrm{h} 24$ hours later. According to these numbers, the expected radiation exposure was estimated during the experiment with the following assumptions:

(i) $10 \mathrm{~cm}$ distance is kept from the target during handling. To achieve this condition, the target is mounted in a cassette.

(ii) It takes 2 minutes for target exchange.

(iii) The dosed target is unloaded 1 hour after irradiation for the ILC mode, while 24 hours after for the KEKB mode.

Under those conditions, the estimated total exposure was $9.9 \times 10^{-2} \mu \mathrm{Sv}$ for the KEKB mode and $3.85 \times$ $10^{-1} \mu \mathrm{Sv}$ for the ILC modes. These numbers are even lower compared to the KEK radiation safety policy, $0.5 \mathrm{mSv} /$ day [13].

\section{EXPERIMENT}

The IPPAK experiment was carried out on 29 and 30 July, 2005, at KEKB. It was started at 16:00 and terminated at 8:00 morning. The first several hours were spent for kicker manipulation to avoid the vertical sweep from bypassing the vertical kicker electrodes and connecting to a dummy load.

In the ILC mode, bunch position adjustment is essential because there is an ambiguity concerning the kicker pulse timing compared to the bucket position. The $1 \mathrm{~mm}$ thick alumina plate, which is usually used to confirm the beam position at the entrance of the beam dump, was replaced with a $100 \mu \mathrm{m}$ thick plate to improve the spatial resolution. A CCD camera, which had been used to investigate the illuminated point by the beam on the plate, was also replaced with one equipped with a 24-times zoom for precise measurements of the positions. In the online monitor for the alumina plate, $5 \mathrm{~mm}$ tick mark drawn on the plate was seen as a $37 \mathrm{~mm}$ one on the display; the effective magnification was 7.4. In horizontal direction, there was an additional magnification of 1.41 due to a 45 -degree incline to the beam axis. Thus, the total magnification was 10.4 (horizontal) and 7.4 (vertical). Assuming a spatial resolution of $2 \mathrm{~mm}$ on the display, the effective resolution is $0.19 \mathrm{~mm}$ (horizontal) and $0.27 \mathrm{~mm}$ (vertical).

The relative bunch position was adjusted by scanning the displacement of the 1st and 6th bunches as a function of 


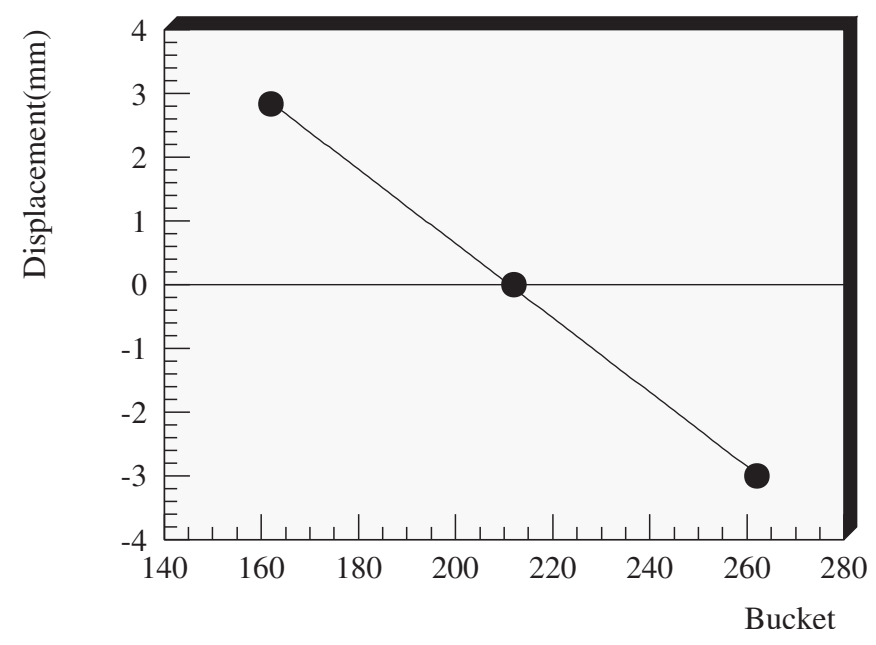

FIG. 3. Displacement between the 1st and 6th bunches in the ILC mode as a function of the relative bucket offset. The displacement is minimized at the expected position where the two bunches fully overlap each other.

the bucket number. The positions of those bunches shift according to the abort-kicker pattern, which is approximated by a sinusoidal curve as a function of the bucket number. The 1st and 6th bunches should move in the contrary directions for a common bucket shift; the difference of their displacements is minimized somewhere. As a result, the displacement was minimized at the expected bucket, as shown in Fig. 3. For the other bunches, in the ILC mode, the position was adjusted one by one in the same manner. The relative bunch position was thus adjusted within the expected spatial resolution, which was sufficient to make an overlapped beam profile in the ILC mode.

After the bunch adjustment, the experiment was started. Table IV summarizes the experimental menu. KEKB390 refers to the KEKB mode, which has a total current of $390 \mathrm{~mA}$ and a bunch charge of $3.0 \mathrm{nC}$. The bunch power of 1 bunch is $3.0 \mathrm{nC} \times 8.0 \mathrm{GeV}=24 \mathrm{~J}$, giving a power density on the target of more than $600 \mathrm{~J} / \mathrm{mm}^{2}$. ILC 0.7 means the ILC mode with single bunch, but the bunch charge is $70 \%$ of the nominal value of $10 \mathrm{nC}$. The bunch charge in ILC1 is $10 \mathrm{nC}$. For ILC2, two bunches were filled with $10 \mathrm{nC}$ charges.

ILC2 was taken twice; totally, 5 targets were examined. All of the shots were taken with W-Re blocks which were
FIG. 4. Beam image taken by developing paper attached upstream of the test target. The tail, which is extended to the lower right direction, is considered to be made by lower energy particles, which scatter with the vacuum window and the air molecules.

$15 \mathrm{~mm}$ thick. The targets were exchanged manually by accessing the accelerator tunnel one hour after each experimental shot, in which the radiological activity reasonably decreased.

The beam position on the block was confirmed by developing papers mounted in front of the block. As shown in Fig. 4, which was taken in the ILC0.7 mode, the beam image was clearly observed for all shots. The exact size of the beam is hard to estimate from the image because the density of the image is not proportional to the amount of beam, but the size is likely to be consistent with the beam size in KEKB. If the size of the core of the dark area corresponds to FWHM of the beam size, the horizontal and vertical beam size (FWHM) are 1.4 and $0.66 \mathrm{~mm}$, respectively. Those numbers correspond to 0.58 and $0.28 \mathrm{~mm}$ in standard deviation, respectively. The beam size in KEKB HER is $0.6 \mathrm{~mm}$ in the horizontal and $0.3 \mathrm{~mm}$ in the vertical directions (standard deviation), but a slight increment is expected by-passing $4 \mathrm{~m}$ long air.

\section{THE SURFACE INVESTIGATION}

After the experiment, several weeks were taken for cooling down the radiological activation, and the surface of the target surface was observed by an optical scope, which had 300 magnification at maximum. Targets had

TABLE IV. Summary of the experimental menu. All of the shots were taken with a $15 \mathrm{~mm}$ thick W-Re block.

\begin{tabular}{lcccc}
\hline \hline & Current $(\mathrm{mA})$ & Density $\left(\mathrm{J} / \mathrm{mm}^{2}\right)$ & Flux $(\mathrm{J} / \mu \mathrm{s})$ & Target ID \\
\hline KEKB390 & 390 & $600-4600$ & 3780 & Target 1 \\
ILC 0.7 & 5 & 280 & 32.8 & Target 4 \\
ILC 1 & 7 & 560 & 65.5 & Target 3 \\
ILC 2 & 14 & 1120 & 131 & Targets 2 and 5 \\
\hline \hline
\end{tabular}




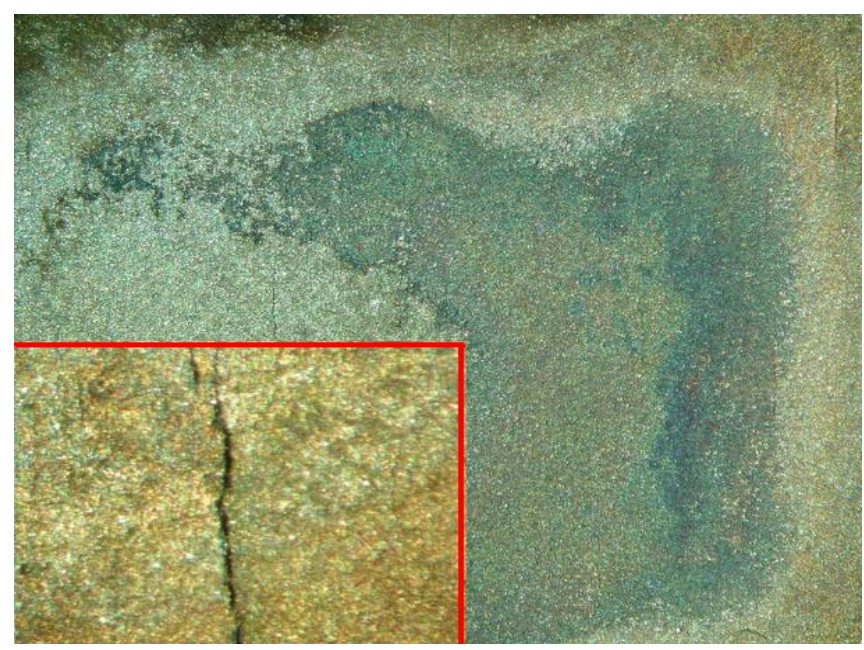

FIG. 5. (Color) Downstream surface of the target dosed with the KEKB390 mode (target 1). The horizontal size of this picture corresponds to $10 \mathrm{~mm}$. As can be clearly seen, the surface color was changed to be dark, like burn out. The picture superimposed on the left-lower corner is an expanded image, where a crack is observed. The horizontal size corresponds to $1 \mathrm{~mm}$.

been wrapped in a piece of thermal paper and mounted in a cassette. A spot on the paper made by beam injection also provided important information.

The target surface dosed with the KEKB390 mode (target 1) is shown in Fig. 5. This surface corresponds to downstream of the beam direction. The color was greatly changed, widely like a burnout. The thermal paper was really burned out at the downstream side. By looking at the surface of the target with much greater magnification,

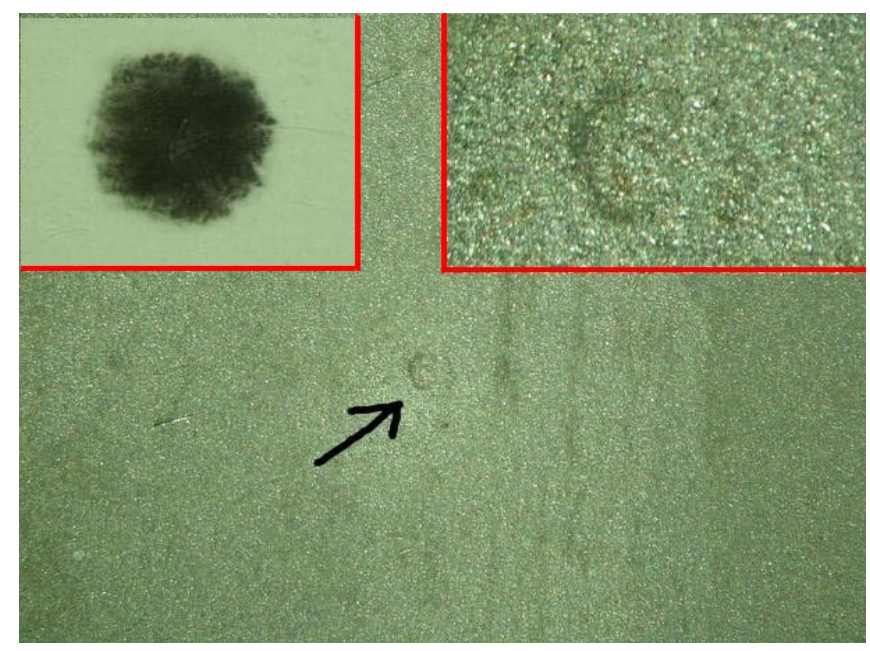

FIG. 6. (Color) Downstream surface of the target dosed with the ILC2 mode (target 2). The horizontal size of this picture corresponds to $10 \mathrm{~mm}$. There is a small colored area that can be identified as the beam trace because a clear black spot was observed at the same position in the thermal paper as shown in the left-upper corner. An expanded image of this spot is superimposed in the right-upper corner.

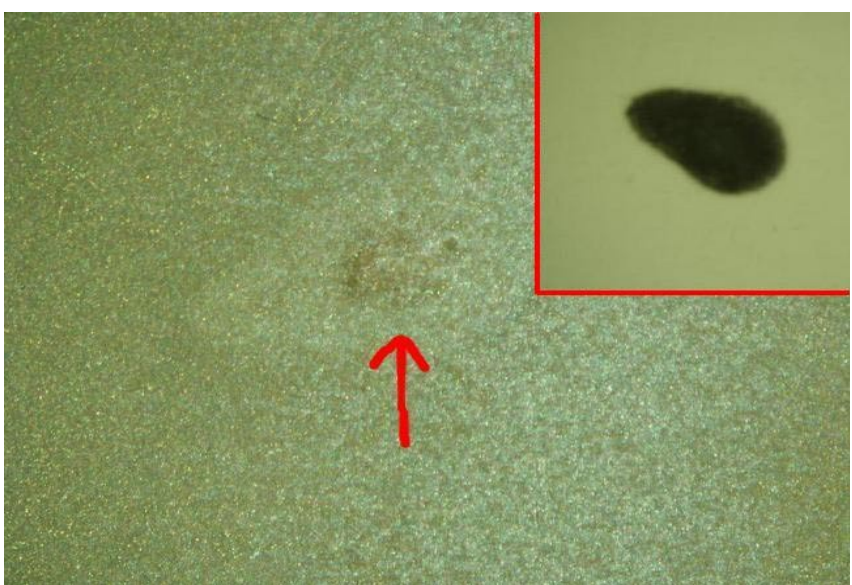

FIG. 7. (Color) Downstream surface of a target dosed with the ILC2 mode (target 5). A beam in the ILC 2 mode was injected twice. The horizontal size of this picture corresponds to $5 \mathrm{~mm}$. There is a small colored area that can be identified as the beam trace because a clear black spot was observed at the same position in the thermal paper, as shown in the right-upper corner.

many cracks were observed, as shown in the superimposed image of Fig. 5. Cracks were also observed in the upstream and side surfaces. The sizes of the cracks were not measured exactly, but they might have been $10 \mu \mathrm{m}$ or less. All cracks were aligned in the same direction. This fact might show that the W-Re block was not isometric.

For the ILC2 mode, two targets (targets 2 and 5) are examined. In target 2, a small colored area was observed downstream, as shown in Fig. 6. In the superimposed image at the upper-right corner, the center spot is expanded. The color of the spot is lighter than that in the KEKB 390 mode.

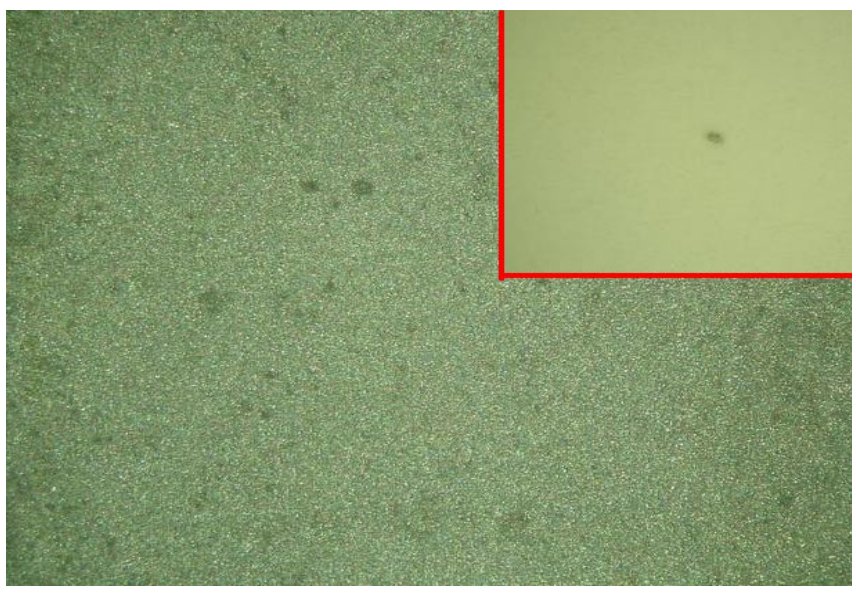

FIG. 8. (Color) Downstream surface of the target dosed with the ILC1 mode (target 3 ). The horizontal size of this picture corresponds to $10 \mathrm{~mm}$. There is an unclear colored spot that can be identified as the beam trace because a very tiny black point was observed at the same position on the thermal paper, as shown in the right-upper corner. In the developing paper, a clear black spot was observed. 


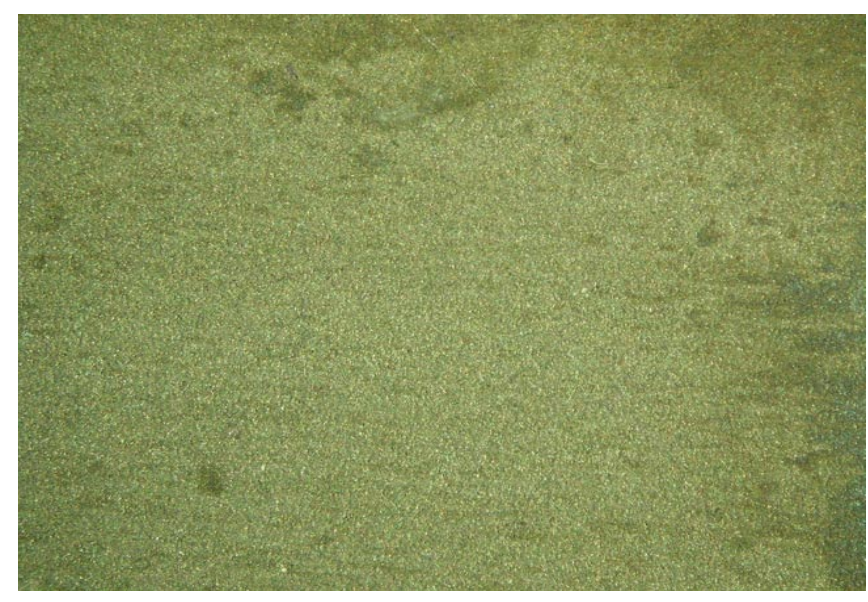

FIG. 9. (Color) Downstream surface of the target dosed with the ILC0.7 mode (target 4). The horizontal size of this picture corresponds to $10 \mathrm{~mm}$. No trace was observed at all on the target surface. There was no indication of changes on the thermal paper. A clear black spot on the developing paper, as shown in Fig. 4, is evidence that the beam passed through the target.

This spot corresponds to the beam path because there is a similar spot on the thermal paper, which is superimposed in the upper-left corner. No cracks were observed at all. Figure 7 shows the downstream surface of target 5. For this target, the ILC2 mode beam was injected twice with an interval of several minutes. The horizontal size of this picture corresponds to $5 \mathrm{~mm}$. There is a colored area similar to that on target 2 , but the black image on the thermal paper is larger than that for target 2, and the shape is not a circle, as shown in the superimposed image at the right-upper corner. The reason for this distorted image on the thermal paper is considered to be heat deposition by the first shot.

For the ILC1 mode, a tiny colored area was observed downstream, but the spot was so unclear that it cannot be found easily, as shown in Fig. 8 (target 3). The spot on the thermal paper was small, as shown in the superimposed image in the upper-right corner. No cracks were observed at all.

In the ILC0.7 mode, no colored area and no cracks were observed in the test target (target 4), as shown in Fig. 9. There were no spots on the thermal paper. Only a development paper set on the front of the target responded to the beam, as shown in Fig. 4.

\section{SUMMARY}

An impressive result was that the damage was varied greatly from that of KEKB390, which was completely damaged, to ILC0.7 which was not damaged at all. Figure 10 summarizes the results in a plane of the energy density $\left(\mathrm{J} / \mathrm{mm}^{2}\right)$ and energy flux $(\mathrm{J} / \mu \mathrm{s})$. The open circles show the data points, which have either no or light effects. The open triangle corresponds to ILC 2, which has a

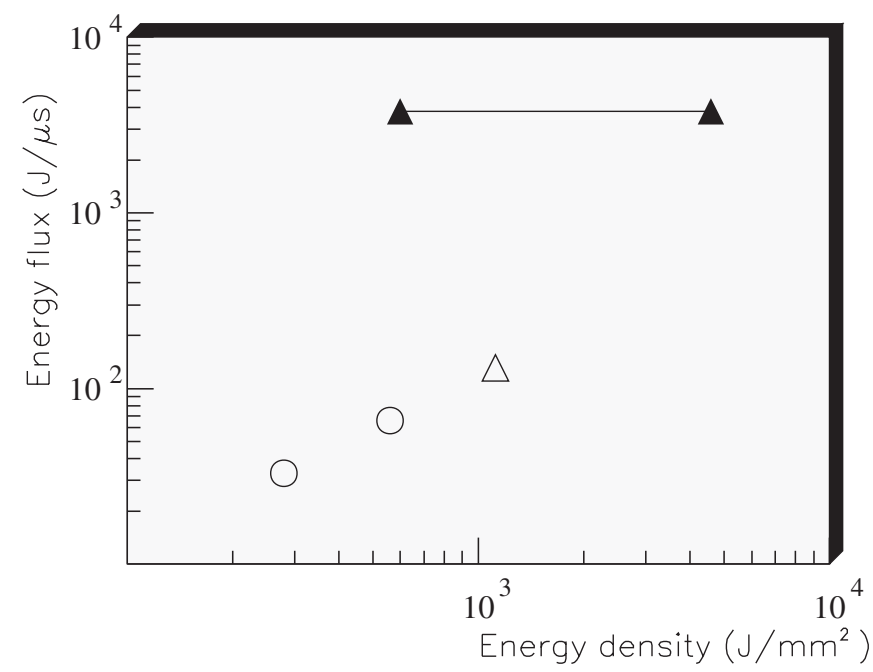

FIG. 10. Results summarized in a plane of the energy density $\left(\mathrm{J} / \mathrm{mm}^{2}\right)$ and energy flux $(\mathrm{J} / \mu \mathrm{s})$. The open circle shows results of ILC 0.7 and ILC 1 . The open triangle shows that of ILC 2. The line with closed triangles at the ends shows the data range of the KEKB 390 mode.

colored area on the target. The line terminated with closed triangles shows the range of KEKB 390 mode, where the target was completely damaged.

As clearly shown in Fig. 10, the data points of both the damaged and not damaged cases are in a same range as the energy density. Therefore, it might show some temporal effect that eases the damage. The damage level increases rapidly as the energy flux increases. There might be some critical threshold near this region that produces the difference in the damage level.

Concerning the impact on the ILC positron production, we can state that the conventional method was confirmed by this result, because targets other than the KEKB390 mode survived, or at least were not broken.

If we can extrapolate the effects observed in IPPAK into that in ILC positron production, with the help of simulations and/or additional experiments, we will be able to make some statement with enough confidence concerning positron production in the ILC. Because the IPPAK examined only sudden effect and no fatigue effect, mean operation period without any fatigue effect as a function of the beam power should be estimated.

For example, if the condition corresponding to the ILC1 mode is acceptable in the ILC positron source, i.e. the possible operation period without any fatigue effect is even long, positron production will be possible with a drive beam of $3.2 \mathrm{nC}, 293 \mathrm{~ns}$ bunch spacing, and $132 \mathrm{~m} / \mathrm{s}$ target rotation, since the energy density and flux made by these conditions are the same as those in the ILC 1 mode. In the conventional method, which was discussed at the last ILC workshop held at Snowmass, Colorado, in 2005 August, those parameters had $3.2 \mathrm{nC}, 308 \mathrm{~ns}$ bunch spacing, and $360 \mathrm{~m} / \mathrm{s}$ target rotation. With these parameters, there will 
be much less damage to the target than that in the ILC-1 mode. The results of the IPPAK experiment suggest that the ILC positron beam can be generated with more relaxed parameters, e.g., shorter bunch spacing and/or less target rotation speed.

\section{ACKNOWLEDGMENTS}

The authors appreciate the KEKB and BELLE groups for their help and cooperation. H. Koiso and Y. Funakoshi made many meaningful suggestions to proceed the experiment. The authors also thank H. Tawara and H. Nakamura of Radiation Science Center of KEK, for their great contributions concerning radiation safety studies. IPPAK would not be possible without their work. At last, the authors are deeply grateful to K. Oide for his prominent leadership to approve IPPAK as a test experiment at KEKB.

[1] http://lcdev.kek.jp/

[2] http://www.fnal.gov/directorate/icfa/
[3] http://www.fnal.gov/directorate/icfa/ ITRP_Report_Final.pdf

[4] S. Ecklund, SLAC-CN-128.

[5] R. Brinkmann, K. Floettmann, J. Rossbach, P. Schmueser, N. Walker, and H. Weise, TESLA Technical Design Report Part II (Accelerator), DESY, 2001.

[6] S. Araki et al., KEK Report No. 2005-60, 2005.

[7] W. Stein et al., in the Workshop on Positron Sources for ILC (http://www.astec.ac.uk/id_mag/ID-Mag_Helical_ ILC_Positron_Production_Workshop.htm), 2005.

[8] KEKB B-Factory Design Report, KEK Report 95-7, 1995.

[9] S. Kurokawa et al., Papers in Special issue: The KEKB Asymmetric B-Factory [Nucl. Instrum. Methods Phys. Res., Sect. A 499, 1 (2003)].

[10] N. Iida et al., in Proceedings of EPAC 2002, pp. 24232425, 2002.

[11] K. Saito and Y. Tawara, "Radiation Safety Issue for IPPAK Experiment at KEKB HER" (Japanese), KEK Radiation Science Center (unpublished), 2005.

[12] K. Saito and Y. Tawara, "Radiological Activation in IPPAK Experiment at KEKB HER" (Japanese), KEK Radiation Science Center (unpublished), 2005.

[13] Radiation Safety Guidance (Japanese), KEK Radiation Science Center, 2004. 